\title{
Propuesta de proyecto de exportación: Limón "sin semilla" o lima persa (Citrus latifolia)
}

Export project propoal: "seedless" lemon or Persian lime (Citrus latifolia)

\author{
Mateos Rocha Roberto Alejandro ${ }^{1 凶}$, Vargas Velázquez Michel Isaías ${ }^{1}$, González Salas Miguel Ángel ${ }^{1}$, \\ González Acosta Alfredo ${ }^{1}$, González Cárdenas Julio César ${ }^{1}$ \\ ${ }^{1}$ Universidad Veracruzana. Facultad de Ciencias Biológicas y Agropecuarias Región Poza Rica- \\ Tuxpan. Licenciatura en Agronegocios Internacionales \\ ${ }^{凶}$ Autor para correspondencia: romateos@uv.mx
}

Recibido: 04/09/2019

Aceptado: 18/11/2019

\begin{abstract}
RESUMEN
México es considerada una economía dinámica y emergente, con una población de 129.2 millones de personas, brinda un enorme potencial para la inversión de las empresas europeas aumentando así sus exportaciones (Secretaría de Economía, 2018). Sin embargo la dependencia económica de Estados Unidos hace que México se adapte a cualquier movimiento en la economía estadounidense afectando de manera negativa la estabilidad de las exportaciones, esto no tiene por qué ser así. La política económica de Estados Unidos que desde el cambio de gobierno se tornó proteccionista obliga a los exportadores mexicanos y del estado de Veracruz principalmente a buscar alternativas de exportación, es ahí donde se plantea un proyecto para exportación a Los Países Bajos como una alternativa viable para exportar el limón persa, así como los costos que generaría el proceso de exportación. Con esto se pretende aportar información que sirva para que los exportadores tengan una opción más y no sigan dependiendo del mercado norteamericano.
\end{abstract}

Palabras clave: Economía, México, Viabilidad.

\begin{abstract}
Mexico is considered a dynamic and emerging economy, with a population of 129.2 million people, it offers enormous potential for the investment of European companies thus increasing their exports (Ministry of Economy, 2018). However, the economic dependence of the United States makes Mexico adapt to any movement in the US economy negatively affecting the stability of exports, this does not have to be this way. The economic policy of the United States that since the change of government became protectionist forces Mexican exporters and the state of Veracruz mainly to seek export alternatives, that is where a project for export to the Netherlands is proposed as a viable alternative for export Persian lemon, as well as the costs that the export process would generate. This is intended to provide information that serves so that exporters have one more option and do not continue to depend on the North American market.
\end{abstract}

Keywords: Economy, Mexico, Viability. 


\section{INTRODUCCIÓN}

Los cítricos se comercializan en su mayoría como producto en fresco y jugo concretado: un gran porcentaje de la comercialización se da en regiones del hemisferio norte, de manera especial en Europa, donde México ocupa el trigésimo lugar; mientras que en las importaciones resaltan naciones como Rusia, China y el bloque europeo así México ocupa el cuadragésimo séptimo lugar.

En lo que a jugo concentrado respecta Brasil junto con EU industrializan un 79\% y México participa con un $9 \%$ de la producción; los principales consumidores de jugo son EU y la Unión Europea; los países exportadores de dicho jugo son Brasil con $74 \%$, México con un $11 \%$ y EU con $7 \%$. El principal estado productor del país es Veracruz seguido de Tamaulipas, Michoacán, San Luis Potosí y Nuevo León, concentrando el $75 \%$ de la producción total.

El rendimiento nacional se ve disminuido si se compara con el mundial donde el promedio en el país es mucho más bajo al global. La producción es consumida en su mayoría en el mercado interno, el restante se exporta a Estados Unidos (Martínez, 2015)

De acuerdo con la secretaría de economía para el 2017 las exportaciones mexicanas de limones sumaron 519 millones de dólares, un aumento de $15 \%$ frente al año previo y un máximo histórico, estas ventas externas escalaron $4 \%$ en 2014 y $24 \%$ en 2015 (Secretaría de Economía 2017).

Ahora bien, en México la producción de limones es económicamente significativa, el limón mexicano se cultiva a lo largo de la costa del pacífico en estados como Michoacán Colima. Guerrero y Oaxaca, mientras que la mayoría de los limones persas se cultivan en un microclima del norte de Veracruz, con menor producción en
Chiapas, Oaxaca, Tabasco, Puebla, Jalisco y Yucatán (Rocha, 2018)

\section{MATERIALES Y MÉTODOS}

Como análisis para esta investigación se planteó una propuesta de exportación que contiene una red de distribución de limón persa de Martínez de la Torre hacia Países Bajos, por vía marítima. Se tomó como referencia a las empresas empacadoras ubicadas en el municipio de Martínez de la Torre, Veracruz.

El destino del embarque fue el continente europeo en el país de Holanda (Países Bajos), dicho embarque será transportado por el modo terrestre y marítimo. El embarque se constituye de un contenedor de 20' que lleva consigo 22 tarimas de limón persa en presentación de 10 lbs con el calibre 42.

Dicha propuesta contiene los siguientes rubros:

$\checkmark$ Distribución de la red logística desde el proveedor de huerta hasta el consumidor final.

$\checkmark$ Descripción de las características de los medios de transporte, así como la documentación necesaria para llevar cabo la exportación del producto

$\checkmark$ Costes para poder realizar la exportación.

$\checkmark$ Tiempos de producción y proceso del producto

$\checkmark$ Tiempos de recorrido del transporte

\section{RESULTADOS}

Considerando todos los aspectos investigados, se obtuvo lo siguiente:

\section{Características del producto a exportar:}

Los frutos maduros de la lima Tahití tienen un contenido de jugo del $40 \%$ al $60 \%$, el jugo tiene 
un índice de acidez del 5 al 6\%, la cantidad de sólidos solubles del 7 al $8 \%$ y un contenido de ácido ascórbico de 20 a $40 \mathrm{mg}$ por $100 \mathrm{ml}$ de jugo. La cáscara del fruto tiene un espesor de 2 a $3 \mathrm{~mm}$, el fruto usualmente tiene una papilla o pezón al final del estilo, variando considerablemente en tamaño y forma (Urbina et al., 2011).

La calidad requerida tanto a nivel nacional como global se distingue conociendo las características principales del limón estas determina estándares establecidos por los países que importan como lo son la apariencia, sabor, tamaño, color y sanidad.

\section{Normatividad nacional e internacional aplicable al limón persa}

En México se debe considerar la normatividad establecida por la Dirección General de Normas, que es quién emite la Norma Oficial Mexicana (Secretaría de Economía, 2015). El Servicio Nacional de Sanidad, Inocuidad y Calidad (SENASICA) deberá verificar, vigilar y supervisar a fin de garantizar que se toman las siguientes medidas:

Limpieza y desinfección.

Medidas de prevención de re-contaminación.

Evitar mezclar fruta de otros mercados con destino a Corea.

Eliminación de contaminantes tales como: hojas, ramas y tierra (suelo) en los envíos de exportación.

(Senasica, 2015).

\section{Inspección}

SENASICA supervisará para garantizar la aplicación de las siguientes medidas. Por cada embarque se tomará una muestra del $2 \%$ de cajas de fruta empacada; Inspección visual de las plagas objetivos (mencionadas) por medio de cortes de la fruta; Inspección especifica en el tallo (pedicelo - pedúnculo) de la fruta para comprobar la presencia de Pantomorus cervinus;
En caso de que cualquier espécimen vivo de las plagas objetivo que se intercepte en el proceso de exportación, quedará prohibido la exportación de Limón Persa a Países Bajos para esa temporada; Así también en caso de encontrar cualquier espécimen vivo enumeradas 63 dentro de las plagas cuarentenarias para Países Bajos durante la inspección, los embarques deben ser rechazados por el SENASICA. Sin embargo, si las plagas se encuentran muertas o totalmente destruidas o eliminadas podrán ser aprobadas por el SENASICA, se notificará a AQSIQ para su conocimiento.

\section{Certificación}

SENASICA expedirá un certificado fitosanitario por cada embarque/contenedor que aprobó la inspección y será descrita en el C.F.: Número de registro de huertos y empaque de exportación; Una leyenda que indica que el envío cumple con los requisitos descritos en el protocolo de requisitos fitosanitarios para la exportación de limón persa desde México a Países Bajos y está libre de plagas de importancia cuarentenaria para Países Bajos.

Norma internacional CODEX STAN 213, será siempre prioritario considerar los lineamientos que se instruyen para el limón persa en el Codex Alimentarius en su Anteproyecto de norma del CODEX para la lima-limón”. En las categorías, de conformidad con las disposiciones especiales para las categorías y las tolerancias permitidas, las limas limones deberán: Estar intactas; Estar firmes; Estar sanas; Deberá excluirse todo producto afectado por podredumbre o que esté deteriorado de tal manera que no sea apto para el consumo; Estar limpias, prácticamente, exentas de materias extrañas visibles; Prácticamente exentas de magulladuras; Prácticamente exentas de plagas y daños causados por plagas; Estar exentas de daños causados por temperaturas bajas; Estar exentas de humedad externa anormal, excepto la condensación posterior a su 
remoción de una cámara frigorífica; Estar exentas de cualquier olor y/o sabores extraños: Estar sin semilla:

Las limas/ limones deberán ser recolectadas con cuidado, y se dará margen a los criterios específicos de la "variedad, el área en que se cultivaron, al desarrollo y a la condición de la maduración debe ser tal" que les permita a los productos: Poder soportar la manipulación y el transporte, y mantenerse en buenas condiciones al llegar al lugar de destino (FAO, 2015).

\section{Contenido Mínimo de Jugo y Coloración}

El contenido mínimo de jugo se calcula con relación al peso de total de la fruta, haciéndose la extracción de manera manual, el cual deberá ser de $42 \%$, y su coloración debe ser normal para la variedad y tipo del área de producción según la comisión del Codex Alimentarius (FAO, 2015).

\section{Embarque}

Se debe considerar que es un embarque de 22 tarimas de limón persa en presentación de 10 lbs con el calibre 42, pero para poder obtener ese cargamento se deben de correr los $58938 \mathrm{k}$ que entran a la empacadora, para poder correr esta cantidad de kilos es necesario un tiempo de 4.13 horas.

Cuadro 2. Tiempo de procesamiento del embarque

\begin{tabular}{|l|l|}
\hline \multicolumn{1}{|c|}{ Actividad } & \multicolumn{1}{c|}{ Tiempo } \\
\hline Arribo de mercancía & $4.13 \mathrm{~h}$ \\
\hline Descanicado & $4.13 \mathrm{~h}$ \\
\hline Preselección & $4.13 \mathrm{~h}$ \\
\hline Lavado & $4.13 \mathrm{~h}$ \\
\hline Cepillado & $4.13 \mathrm{~h}$ \\
\hline Presecado & $4.13 \mathrm{~h}$ \\
\hline Encerado & $4.13 \mathrm{~h}$ \\
\hline Seleccionado & $4.13 \mathrm{~h}$ \\
\hline Secado & $4.13 \mathrm{~h}$ \\
\hline Calibrado & $4.13 \mathrm{~h}$ \\
\hline Empacado & $4.13 \mathrm{~h}$ \\
\hline Estibado & $4.13 \mathrm{~h}$ \\
\hline Paletizado & $4.13 \mathrm{~h}$ \\
\hline Embarcado & $4.13 \mathrm{~h}$ \\
\hline
\end{tabular}

Fuente: Elaboración propia con datos de Comfruver, 2019. 
Proceso de empacado de limón persa:

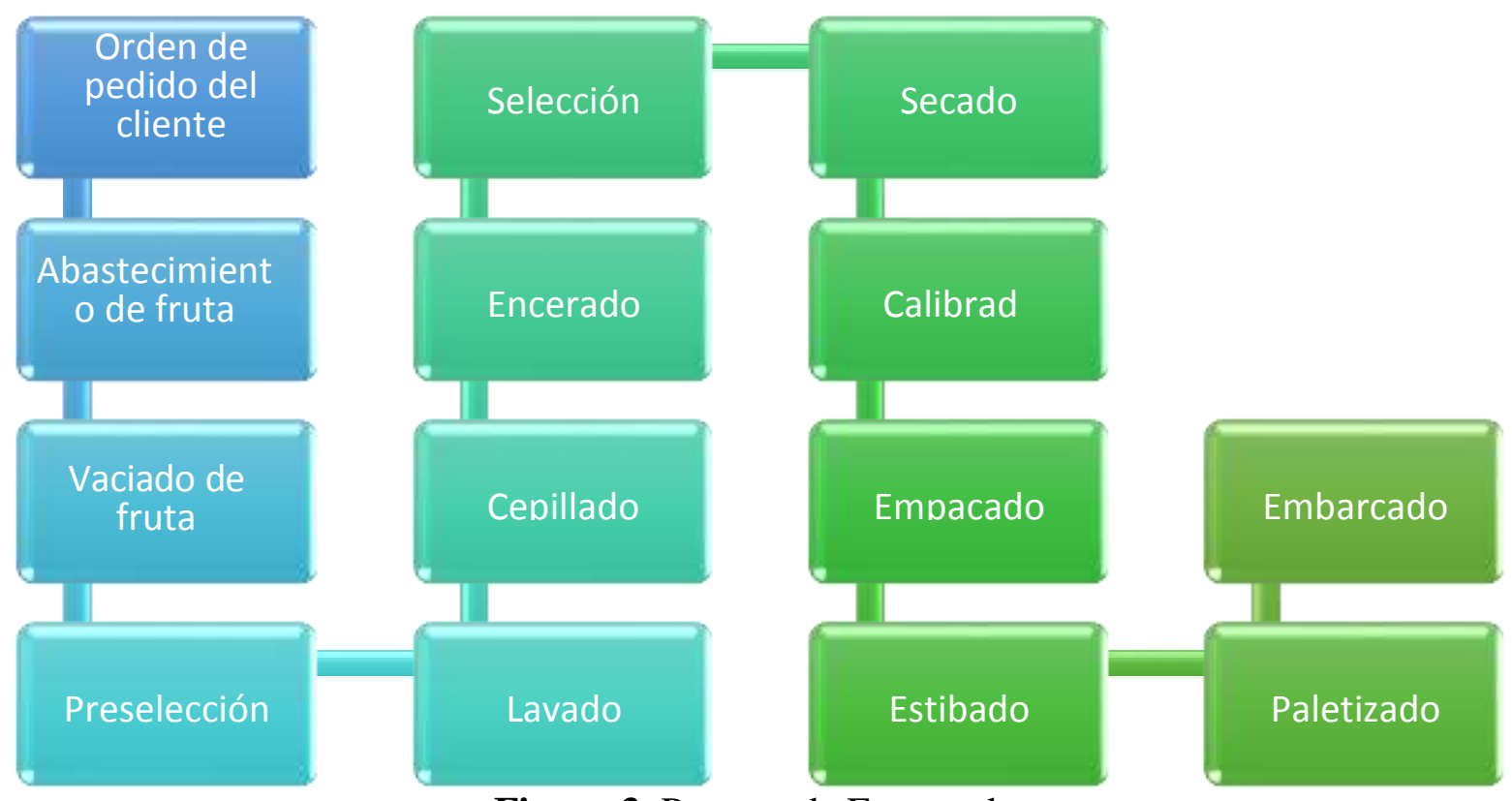

Figura 3. Proceso de Empacado

Fuente: Elaboración propia con datos de Veca Produce, 2019.

\section{Suministro de materia prima requerida}

Para poder realizar un embarque de limón de 10 libras se necesita un promedio de 22 pallets, estos están divididos de la siguiente manera. Cada pallet se constituye por 228 cajas de 10 libras, estas 228 cajas son divididas en 19 camas de 12 cajas a su vez. El peso promedio de cada caja de 10 libras es 4.53592 kilogramos.

Por lo que para poder a completar un embarque de 10 lbs se necesitan $22 * 228 * 4.53592=22,753$ kilogramos redondeando la cantidad en kilos.

Se debe considerar que no toda la materia prima que llega a la empacadora es calidad verde para exportar a Países Bajos, por lo tanto, un estimado en volumen se requiere un aproximado de 58938 $\mathrm{kg}$ de los cuales al correrlos y clasificarlos se estará obteniendo los 22,753 kg de limón verde para poder exportar a Países Bajos.

Con esto se requieren otros insumos como lo son el cartón del cual se requieren 228 cajas con su respectiva tapa, además de 22 tarimas, nylon para emplayar, flejes esquineros. También se debe de contar con otros insumos como son agua, cera, energía eléctrica, transporte y mano de obra.

\section{Contenedor marítimo}

Como el embarque se transportará por vía terrestre y vía marítima se necesita que la carga sea manipulada lo menos posible, es por ello por lo que se debe utilizar un contenedor marítimo ya que será transportado por la vía marítima. Las características del contenedor marítimo son las siguientes 


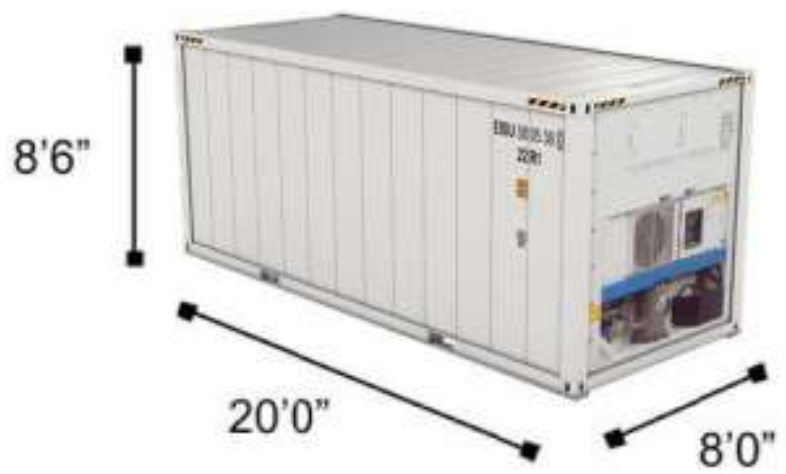

Figura 4. Contenedor Reefer.

Fuente: Iberforwrders, 2019.

\section{Rutas terrestres opcionales}

Se tienen dos opciones para mandar el producto a Países Bajos, las rutas son Martínez de la Torre -Altamira o Martínez de la Torre - Puerto de Veracruz. De Martínez de la Torre a Altamira es un viaje de 5 horas con 5 minutos tal como lo indica el mapa, además tendría que viajar 339 kilómetros para llegar a su destino. La ruta más ideal sería Martínez de la torre a Poza Rica, de Poza Rica a Tuxpan y de Tuxpan a Tampico hasta llegar a Altamira.

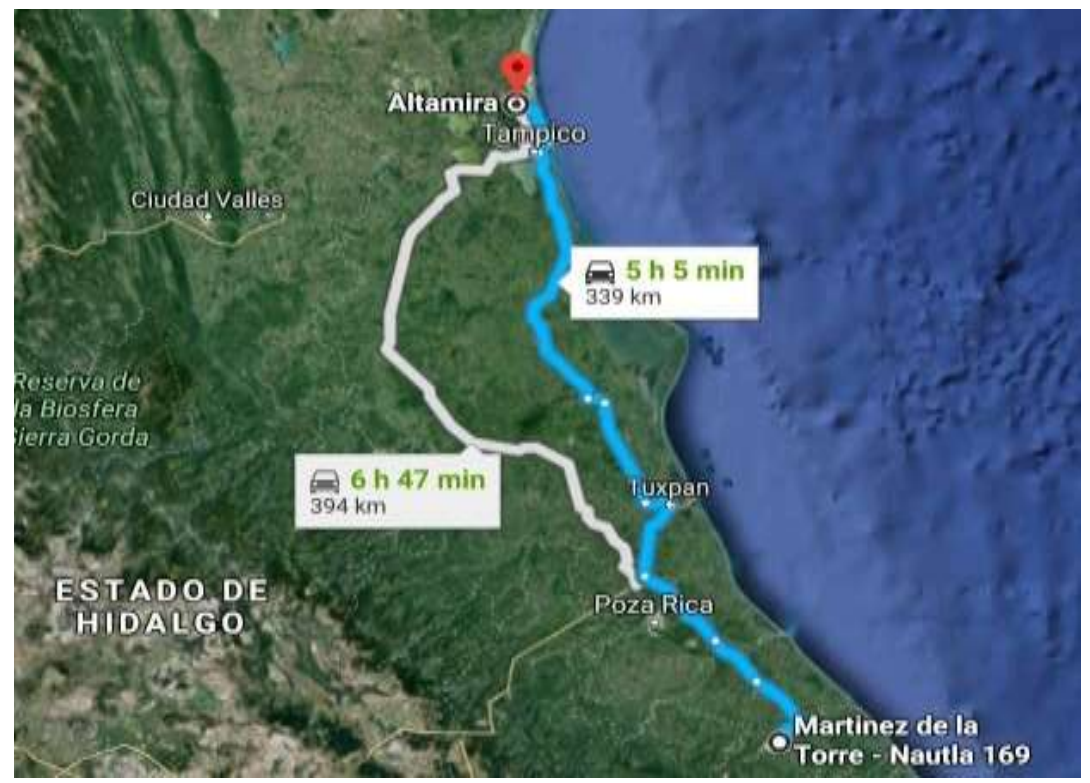

Figura 5. Ruta Martínez- Altamira

Fuente: Elaboración propia con datos de Costa de Veracruz, 2019.

De Martínez de la Torre al puerto de Veracruz el tiempo de viaje sería de 3 horas con 15 minutos con un total de 195 kilómetros por recorrer. La ruta más ideal sería de Martínez de la Torre a Vega de Alatorre y de Vega de Alatorre al Puerto de Veracruz. 


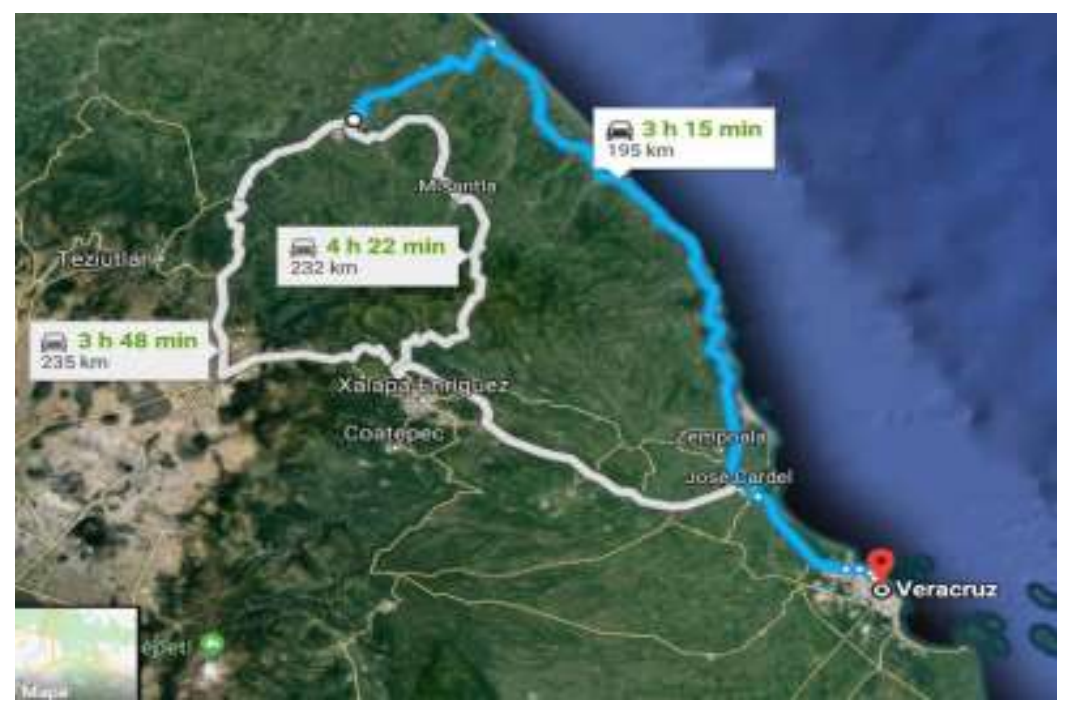

Figura 6. Ruta Martínez - Veracruz

Fuente: Elaboración propia con datos de Costa de Veracruz, 2019.

Para lo anterior se realizaron dos diferentes cotizaciones quedando de la siguiente manera para la vía de Martínez de la Torre - Veracruz (Contenedor). Estos costos se pagarán dependiendo los días de anticipación que se requiera el contenedor en puerto. (Alrededor de $\$ 600$ a $\$ 1,000$ pesos por contenedor por día).

Cuadro 3. Vía Martínez de la Torre - Veracruz (Contenedor)

\begin{tabular}{|c|c|c|c|c|}
\hline \multicolumn{2}{|l|}{ Actividad } & \multicolumn{3}{|c|}{ Costo por contenedor } \\
\hline \multicolumn{2}{|l|}{ Maniobra de recolección de contenedor en patio de vacios } & \multicolumn{3}{|c|}{1111.35} \\
\hline \multicolumn{2}{|c|}{ Flete de contenedor Veracruz - Martínez de la Torre - Veracruz } & \multicolumn{3}{|c|}{10455} \\
\hline \multicolumn{2}{|l|}{ Maniobra de descarga de contenedor en puerto } & \multicolumn{3}{|c|}{1960.24} \\
\hline & Total & $\begin{array}{l}\$ \\
13,526\end{array}$ & & \\
\hline Costos Adicionales en puerto de Veracruz & \multicolumn{2}{|c|}{$20^{\circ}$} & & $40^{\circ}$ \\
\hline $\begin{array}{l}\text { Energia Eléctrica para contenedores refrigerados } \\
\qquad \text { por dia o fracción * }\end{array}$ & $\$$ & 327.76 & $\$$ & $1,483.70$ \\
\hline
\end{tabular}

Fuente: Elaboración propia con datos de Comfruver, 2019.

Para el caso de la vía Martínez de la Torre Altamira (Contenedor). En Altamira también se pagará conexión de contenedores los días requeridos en base a los días que solicite la naviera el contenedor en puerto para su embarque. (Alrededor de $\$ 600$ a $\$ 1,000$ pesos por contenedor por día). 
Cuadro 4. Vía Martínez de la Torre - Altamira (Contenedor)

\begin{tabular}{|c|c|}
\hline Actividad & Costo por contenedor \\
\hline Maniobra de recolección de contenedor en patio de vacios & $\$ 600$ \\
\hline Flete de contenedor Altamira - Martínez de la Torre - Altamira & $\$ 12,640$ \\
\hline Maniobra de descarga de contenedor en puerto & $\$ 1,500$ \\
\hline Total & $14,740.00$ \\
\hline
\end{tabular}

Fuente: Elaboración propia con datos de Comfruver, 2019

\section{Cotizaciones de rutas marítimas}

Las cotizaciones se realizaron con Conteiners para las dos rutas marítimas

\begin{tabular}{|c|c|}
\hline PUERTO DE ORIGEN & ALTAMIRA \\
\hline PUERTO VIA & Directo \\
\hline PUERTO DE DESTINO & ROTTERDAM \\
\hline TIEMPO DE TRÁNSITO ESTIMADO & 21 Días \\
\hline FREC. & 7 Dias \\
\hline FECHA DE SALIDA & 14-ago-2019 \\
\hline CONTENEDORES & $1 \times D V 20$ \\
\hline SEGURO & No \\
\hline INCOTERM & - \\
\hline Desglose de precios & 人 \\
\hline FLETE $(M \times)$ & $7.659,61 \mathrm{MXN}$ \\
\hline THC PUERTO LOCAL (MX) & $9.564,76 \mathrm{MXN}$ \\
\hline $\mathrm{B} / \mathrm{L}$ ISSUANCE (MX) & $1.464,00 \mathrm{MXN}$ \\
\hline DESPACHO DE ADUANAS (MX) & $4.879,98 \mathrm{M} \times \mathrm{N}$ \\
\hline $\begin{array}{l}\text { ENVIO B } / \text { L EN PAPEL (NACIONAL) } \\
(M \times)\end{array}$ & $1.073,60 \mathrm{MXN}$ \\
\hline AMS $(M X)$ & $1.464,00 \mathrm{MXN}$ \\
\hline $\begin{array}{l}\text { ENVIO B/L EN PAPEL } \\
\text { (INTERNACIONAL) }(M X)\end{array}$ & $1.073,60 \mathrm{MXN}$ \\
\hline SOLAS VGM PESAJE (MX) & $1.171,20 \mathrm{MXN}$ \\
\hline TOTAL & $50,75 \mathrm{MXN}$ \\
\hline
\end{tabular}

Figura 7. Cotización Altamira Fuente: Conteiners, 2019.



Figura 8. Cotización Veracruz Fuente: Conteiners, 2019.

\section{Análisis de las diferentes vías marítimas}

Tiempo de transporte de Martínez de la Torre al puerto de Veracruz: 3 horas 15 minutos.

Tiempo de transporte de Martínez de la Torre al puerto de Altamira: 5 horas 5 minutos.
Cabe mencionar que la empresa no cuenta con una flotilla de tráileres por lo que el transporte es subcontratado, es por ello por lo que se cotizaron con las diferentes navieras y líneas transportistas de tracto camiones y contenedores marítimos. 
Cuadro 5. Comparativo del costo en las diferentes vías.

\begin{tabular}{|c|c|c|c|c|c|c|}
\hline Origen & Destino & $\begin{array}{l}\text { Costo de } \\
\text { envío al } \\
\text { puerto }\end{array}$ & $\begin{array}{l}\text { Medio } \\
\text { utilizado }\end{array}$ & $\begin{array}{l}\text { Costo de envío } \\
\text { al puerto de } \\
\text { Róterdam }\end{array}$ & Medio utilizado & Total \\
\hline $\begin{array}{c}\text { Martínez de la } \\
\text { torre }\end{array}$ & $\begin{array}{l}\text { Puerto de } \\
\text { Veracruz }\end{array}$ & $\$ 14,854.35$ & $\begin{array}{l}\text { Tráiler con } \\
\text { contenedor } \\
\text { marítimo }\end{array}$ & $\$ 28,350.75$ & $\begin{array}{c}\text { Barco } \\
\text { portacontenedores }\end{array}$ & $\$ 43,205$ \\
\hline $\begin{array}{c}\text { Martínez de la } \\
\text { torre }\end{array}$ & $\begin{array}{c}\text { Puerto de } \\
\text { Altamira } \\
\text { Tamaulipas }\end{array}$ & $\$ 16,067.76$ & $\begin{array}{c}\text { Tráiler con } \\
\text { contenedor } \\
\text { marítimo }\end{array}$ & $\$ 28,350.75$ & $\begin{array}{c}\text { Barco } \\
\text { portacontenedores }\end{array}$ & $\$ 44,418.5$ \\
\hline
\end{tabular}

Fuente: Elaboración propia con datos de Comfruver y Conteiners, 2019.

\section{Requerimientos para exportación}

El cítrico para su empaque requiere de un estricto control de calidad a fin de reunir las características necesarias para su exportación. Los productores para realizar el proceso de cosecha deben tener un especial cuidado en el corte de la fruta fin de disminuir los efectos o alteraciones de la cáscara de la fruta. Para su empaque se utilizan cajas de cartón en sus diferentes presentaciones de acuerdo con el mercado destino.

Los tamaños que requiere para su empaque se clasifican de la siguiente manera

Cuadro 6. Calibres según mercado destino

\begin{tabular}{|l|l|}
\hline \multicolumn{1}{|c|}{ EMPAQUE } & \multicolumn{1}{c|}{ CALIBRE } \\
\hline $\mathbf{4 0}$ libras (USA CANADA, Corea) & $110,150,175,200,230$ y 250. \\
\hline $\mathbf{1 0}$ libras (USA, UE) & $42,48,54$ y 63 \\
\hline $\mathbf{5}$ kilos (Japón y Corea del Sur) & 40 y 44 \\
\hline $\mathbf{2}$ kilos (Japón) & 20 y 24 \\
\hline $\mathbf{1}$ libra (USA, CANADA, UE) & 200 s y 230 s \\
\hline
\end{tabular}

Fuente: Cítricos Cadillo, 2018.

Es importante puntualizar que la fruta que adquiere la empresa debe cumplir con las siguientes especificaciones indispensables para su exportación:

El limón persa debe ser entregado entero y de aspecto fresco.

Limpio y sano.

Bien desarrollados (tamaño adecuado).

De consistencia firme y textura uniforme.
De forma, sabor y olor característico.

Prácticamente exentos de magulladuras y grietas.

Exentos de cualquier olor y/o sabor extraño.

Exentos de materia extraña visible (tierra, manchas o residuos de materia orgánica). Exentos de plagas o de daños causados por las mismas (picaduras de insectos).

Libres de heridas sin sanar. 
Libres de daño causado por las uñas de los cosechadores o personal de empaque.

Libres de pudrición.

Libres de piel dura o seca.

Libres de manchas por contacto o de aceite

(oleocelosis).

Libres de escamas, costras o roña.

Libres de enfermedades.

Libres de defectos mecánicos, meteorológicos, genético- fisiológico, los ocasionados por

plagas, enfermedades u otros.

Color verde oscuro. $70 \%$ para Estado Unidos
Color verde oscuro. 80\% para Europa

Color verde oscuro. 90\% para Asia

Se excluirá toda fruta afectada por pudrición o deterioro, al grado que no sea permitida para su consumo.

En tamaños y medidas, la fruta deberá cumplir con lo siguiente en función de su mercado destino:

Cuadro 7. Tamaño según mercado destino

\begin{tabular}{c|c|c|c|c}
\hline TAMANO & MM-MM & EE.UU & EUROPA & ASIA \\
\hline 1 & $61-63$ & 110 'S & & 36 \\
\hline 2 & $59-61$ & 150 'S & 42 & 40 \\
\hline 3 & $56-59$ & $175 ' S$ & 48 & 44 \\
\hline 4 & $54-55$ & $200 ' S$ & 54 & 48 \\
\hline 5 & $52-53$ & $230 ' S$ & 54 & 54 \\
\hline 6 & $50-51$ & 250 'S & 63 & \\
\hline
\end{tabular}

Fuente: Cítricos Cadillo, 2018.

\section{Incoterm seleccionado: CIP}

Se decidió emplear el incoterm CIP ya que se trata de un transporte internacional de limón persa enviado desde el Puerto de Veracruz hacia Países Bajos. Algunas de las principales características que distinguen a este tipo de incoterm son las siguientes:

La entrega la realiza el vendedor una vez que la mercancía se pone a disposición del portador contratado por él

Los costes de flete marítimo o aéreo deberán ser pagados por el exportador, para llevar al destino convenido la mercancía, el despacho de exportación recaerá a su cargo mientras que en la prima de seguro contratada por el exportador, los riesgos recaen sobre el importador.

Tras la entrega de los bienes el comprador asume los riegos y costes que de estos deriven.
En caso de la subcontratación de transportistas después de la llegada de la mercancía al punto convenido de destino, el riesgo se transmitirá una vez que la mercancía sea transferida al primer porteador.

¿De qué se hará cargo el exportador? Entrega de la mercancía en su almacén. Carga de la mercancía en el camión. Transporte local en origen desde almacén a puerto.

Despacho de aduana de exportación.

Contratación del seguro de transporte.

Carga y estiba de la mercancía en el buque.

Transporte internacional de la mercancía.

Entrega de la mercancía y documentos necesarios.

Empaquetado y embalaje.

Transporte interior en el país de origen.

Despacho de aduanas en origen.

Flete marítimo internacional. 
Seguro.

¿De qué se hará cargo el importador?

Descarga de la mercancía.

Despacho de aduanas de importación.

Transporte local en destino.

Entrega en el almacén del comprador.

Pago de la mercancía.

Gastos de llegada.

Despacho de aduanas en destino.

Transporte interior en el país de destino.

Pago de aranceles.

\section{Tecnología y actividades empleadas en el tracking}

Seguimiento mediante GPS. Garantizar los envíos para que sean más seguros y la entrega de estos en tiempo y forma hacen que el seguimiento del envío sea esencial para identificar cualquier retraso o perdida que pudiera llevar a la insatisfacción del cliente, por lo que el rastreo de la carga es primordial siendo crucial en la logística así se optó por el uso del GPS para seguir el embarque en tiempo real hasta el puerto destino en Países Bajos. Por lo que desde el momento que la carga de limón persa salga de la aduana mexicana se rastreará minuto a minuto cuál es su ubicación para poder cumplir en tiempo y forma con lo acordado.

\section{CONCLUSIÓN}

El desarrollo del anterior proyecto se describió la distribución de la red de logística para él envió de embarque de limón persa con destino final Países Bajos el punto de partida es la ciudad de Martínez de la torre Veracruz, donde se especificó el tiempo para su producción embarque proceso de empacado mediante un diagrama de flujo, suministro de materia prima requerida, características de los medios de transportes, documentación necesaria para su transportación así también los costos generados, cotizaciones etc.

Se determinó que la ruta óptima es la de Martínez de la Torre con destino al puerto de Veracruz de ahí partirá al puerto de Róterdam en Países Bajos. El tiempo de recorrido es menor que al puerto de Altamira, con las cotizaciones se logró determinar que los costos de mandarlo por el puerto de Veracruz con dirección a Países Bajos son menores.

Se especifican los documentos que se necesitan para poder realizar un embarque de limón persa en presentación de 10 libras con destino al continente europeo, cosa muy particular que cada documento se relaciona el uno con el otro.

Finalmente se mostraron cuáles son las tecnologías que se podrían aplicar para hacer un rastreo efectivo y en tiempo real del embarque para con ello tener la total certeza de que llegará a su destino, además se mostró cuáles son los acuerdos (incoterm) entre proveedor y cliente, para con ello asegurarse de recibir lo acordado entre ambas partes.

\section{LITERATURATURA CITADA}

FAO. (2015). Proyecto de Norma del Codex para la Lima-Limón. 09/0772019, de FAO Sitio web: http://www.fao.org/3/w7169s/w7169s08. $\underline{\mathrm{htm}}$

Martínez M. (2015). Sector alimentario, segundo generador de divisas en el país. 09/03/2019, de El Economista Sitio web: https://www.eleconomista.com.mx/empre sas/Sector-alimentario-segundogenerador-de-divisas-en-el-pais20151016-0074.html

Rocha M. (2018). El cultivo de los Cítricos. INIFAP, 1, 485. 
Mateos et al., 2019

Secretaría de Economía. (2015). Dirección

General de Normas. 07/07/2019, de

Secretaría de Economía Sitio web: 2012-

2015.economia.gob.mx/conoce-lase/atencion-ciudadana/procesosadministrativos/dgn.

Secretaría de Economía. (2017). Cadenas globales de valor: un modelo para la integración de empresas mexicanas. 05/03/2019, de ProMéxico Sitio web: https://promexico.mx/documentos/bibliot eca/cadenas-globales-de-valor.pdf

Secretaría de Economía. (2018). TLC México Unión Europea. 06/01/2019, de Secretaría de Economía Sitio web: http://www.bruselas.economia.gob.mx/sw b/swb/bruselas/TLC_Mex_UE

Senasica. (2015). Programa fitosanitario de apoyo a exportaciones. 03/06/2019, de Senasica Sitio web: http://www.cesavejal.org.mx/divulgacion/ exportacionc/ProtocoloMexicoCore a.pdf

Urbina J. Urbina L. (2011). Limón persa. En Evaluación de la cadena logística en frio para productos perecederos de la zona de Martínez de la Torre Veracruz (86). Altamira, Tamaulipas: ICEST.

Copyright (c) 2019 Roberto Alejandro Mateos Rocha, Michel Isaias Vargas V elázquez, Miguel Ángel González Salas, Alfredo González Acosta y Julio César G onzález Cárdenas

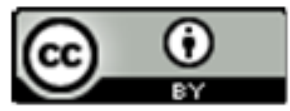

Este tex to está protegido por una licencia licencia Creative Commons 4.0.

Usted es libre para Compartir — copiar y redistribuir el material en cualquier medio o form ato- y Adaptar el documento — remezclar, transformar y crear a partir del material- para cualquier propósito, incluso para fines comerciales, siempre que cumpla la condición de:

Atribución: Usted debe dar crédito a la obra original de manera adecuada, proporcionar un enlace a la licencia, e indicar si se han realizado cam bios. Puede hacerlo en cualquier form a razonable, pero no de form a tal que sugiera que tiene el apoyo del licenciante o lo recibe por el uso que hace de la obra.

Resumendelicencia - Textocompletodelalicencia 